\title{
OPTICAL CHARACTERIZATION OF ERBIUM DOPED III-NITRIDES PREPARED BY METALORGANIC MOLECULAR BEAM EPITAXY
}

\author{
U. Hömmerich*,+, J. T. Seo*, Myo Thaik*, J. D. MacKenzie**, C. R. Abernathy**, S.J. Pearton**, R.G. \\ Wilson†, J. M. Zavada $\ddagger$ \\ *Hampton University, Department of Physics, Research Center for Optical Physics, Hampton, VA 23668 \\ ${ }^{* *}$ University of Florida, Department of Materials Science and Engineering, Gainesville, FL 32611 \\ ${ }^{\dagger}$ Consultant, Stevenson Ranch, CA 91381 \\ ${ }^{\ddagger}$ U.S. Army European Research Office, London, UK, NW1 5 \\ +E-mail: hommeric@gprc.hamptonu.edu
}

\begin{abstract}
We are currently engaged in a systematic study of the optical properties of Er doped IIInitrides prepared by metalorganic molecular beam epitaxy (MOMBE). Under below-gap excitation it was observed that GaN: Er samples with [O] 10 $0^{20} \mathrm{~cm}^{-3}$ and $[\mathrm{C}] \sim 10^{21} \mathrm{~cm}^{-3}$ luminesce at $1540 \mathrm{~nm}$ with an intensity of more than two orders of magnitude greater than samples with low oxygen and carbon concentrations $\left(<10^{19} \mathrm{~cm}^{-3}\right)$. Associated with the different oxygen and carbon concentrations were different thermal quenching behaviors and below-gap absorption bands. Interestingly, for above-gap excitation only small differences in absolute $\mathrm{Er}^{3+} \mathrm{PL}$ intensity and quenching behavior were observed for samples of varying $\mathrm{O}$ and $\mathrm{C}$ content. Initial lifetime studies were performed and showed a rather unusual short decay time of $\sim 100 \mu$ s at room temperature. In order to gain more insight in the $\mathrm{Er}^{3+} \mathrm{PL}$, a comparison of the integrated PL intensity and lifetime was performed for the temperature range $15-500 \mathrm{~K}$. The result reveals that the $\mathrm{Er}^{3+} \mathrm{PL}$ quenches above room temperature due to the onset of non-radiative decay and the reduction in excitation efficiency. All samples were also investigated for visible luminescence. Red luminescence was observed from $\mathrm{GaN}$ : $\mathrm{Er}$ on sapphire substrates under below-gap excitation.
\end{abstract}

\section{INTRODUCTION}

Rare earth doped semiconductors have been studies for more than a decade because of the possibility to develop compact and efficient electroluminescence devices. ${ }^{1,2,3,4}$ Trivalent Erbium ions $\left(\mathrm{Er}^{3+}\right)$ are of special interest because they exhibit an atomic-like transition at 1540nm, which corresponds to the low-loss window of silica-based optical fibers. Electroluminescence devices based on $\mathrm{Er}$ doped $\mathrm{Si}$ and GaAs were reported, however, their efficiency was too low for practical applications. ${ }^{3,4}$ An important step forward in the field of Er doped semiconductors was the observation that the room-temperature $\mathrm{Er}^{3+}$ photoluminescence (PL) intensity strongly depends on the bandgap of the host materials. It was found that for larger bandgap energy, there is less detrimental temperature quenching of $\mathrm{Er}^{3+} \mathrm{PL}$ occurring. ${ }^{5,6}$ Therefore, doping $\mathrm{Er}^{3+}$ ions into wide gap semiconductors is a promising approach to overcome the thermal quenching of $\mathrm{Er}^{3+}$ PL. Consequently, current research efforts focus on studying $\mathrm{Er}^{3+}$ doped into wide bandgap semiconductors such as $\mathrm{SiC}$ and $\mathrm{GaN}^{7,8}$ First results on the observation of $1.54 \mu \mathrm{m}$ photoluminescence from $\mathrm{Er}$ implanted $\mathrm{GaN}$ and $\mathrm{AlN}$ were reported by Wilson et al. ${ }^{9}$ Subsequently, other research groups reported results of photoluminescence, cathodoluminescence, and electroluminescence (EL) studies of Er implanted GaN. ${ }^{10,11,12,13}$ The goal for practical device applications is to incorporate $\mathrm{Er}^{3+}$ ions during epitaxial growth. Doping during growth has several advantages over ion implantation including the absence of implantation damage, unlimited layer thickness, and a more homogenous doping profile. In-situ 
Er doping of AlN using metalorganic molecular beam expitaxy (MOMBE) was first reported by MacKenzie et al. in 1996. ${ }^{14}$ This work was followed by the first demonstration of in-situ Er doping of GaN. ${ }^{15}$ Recently, Hansen et al. reported results of in-situ doped GaN: Er prepared by hydride vapor phase growth. ${ }^{16}$ Visible PL and EL from GaN: Er grown by molecular beam epitaxy have been reported. ${ }^{17,18}$ Even though encouraging strong $\mathrm{Er}^{3+} \mathrm{PL}$ results were obtained, initial infrared electroluminescence studies of $\mathrm{GaN}$ : Er have shown rather poor efficiencies. ${ }^{19,20}$ In order to optimize the material preparation for developing more efficient $1.54 \mu \mathrm{m}$ devices, it is crucial to understand the incorporation and excitation mechanisms of $\mathrm{Er}^{3+}$ ions in III-Nitrides. More specifically, it will be important to identify the $\mathrm{Er}^{3+}$ sites that are excited efficiently through electrical injection of electron-hole pairs and to optimize their concentration in the GaN host. ${ }^{12}$ In this paper we present new spectroscopic results focusing on the $1.54 \mu \mathrm{m}$ PL from MOMBE grown Er doped GaN. Results of temperature dependent PL intensity, lifetime, and excitation studies will be shown and discussed for samples with varying oxygen and carbon content. In addition, we will also report some initial results on visible luminescence studies.

\section{EXPERIMENTAL PROCEDURES}

The Er doped GaN films were grown by MOMBE in an INTEVAC Gas Source Gen II on Inmounted (100) $\mathrm{Si}$ or (0001) Sapphire substrates. The GaN films were preceded by a low temperature AlN buffer $\left(\mathrm{T}_{\mathrm{g}}=425^{\circ} \mathrm{C}\right)$. A $0.2 \mu \mathrm{m}$ undoped $\mathrm{GaN}$ spacer was deposited prior to the growth of GaN: Er. Triethylgallium (TEGa), Dimethylethylamine alane (DMEAA), and thermally-evaporated $8 \mathrm{~N} \mathrm{Ga}$ metal provided the group III fluxes. A shuttered effusion oven with 4N Er was used for solid source doping. Reactive nitrogen species were provided by a SVT radio frequency plasma source. Due to the incorporation of carbon and oxygen from residual ether in TEGa, the $\mathrm{C}$ and $\mathrm{O}$ background observed in TEGa derived GaN were $\sim 10^{21} \mathrm{~cm}^{-3}$ and $\sim 10^{20} \mathrm{~cm}^{-3}$, respectively, as determined by SIMS measurements. GaN grown using thermally-evaporated $8 \mathrm{~N}$ pure $\mathrm{Ga}$ as the group III source had oxygen and carbon backgrounds of less than $10^{19} \mathrm{~cm}^{-3}$. $^{21}$

PL measurements were performed using either the broad-band visible $(457-514 \mathrm{~nm})$ or uv (333.6-363.8nm) outputs of an argon-ion laser for excitation. The luminescence spectra were recorded using a 1-m monochromator equipped with a liquid-nitrogen cooled Ge detector. Visible PL studies employed a thermo-electric cooled photomultiplier tube. The signal was processed using lock-in technique. The samples were cooled on the cold-finger of a two-stage closed-cycle refrigerator. High temperature PL studies were carried out using a homemade heating element, which had a temperature accuracy of $\pm 5^{\circ} \mathrm{C}$. Luminescence lifetime data were taken with a fast response Ge detector and averaged using a digitizing oscilloscope. PLE studies were carried out using an argon pumped dye laser or and a Nd: YAG/Optical Parametric Oscillator system.

\section{RESULTS AND DISCUSSION}

\section{a) $\mathrm{Er}^{3+} \mathrm{PL}$ intensity and spectra}

Figure 1 shows the room-temperature, infrared PL spectra of Er doped $\mathrm{GaN}$ on $\mathrm{Al}_{2} \mathrm{O}_{3}$ prepared by MOMBE. No significant spectral differences were observed for Er doped GaN on Sapphire or Si substrates. For comparison, the $\mathrm{Er}^{3+} \mathrm{PL}$ spectra of AlN: Er (MOMBE) and Er implanted GaN are also shown in figure 1. ${ }^{9,14}$ All samples were excited with the 363-366.8nm UV output of an Argon-Ion laser. UV excitation was chosen to simulate carrier-mediated $\mathrm{Er}^{3+}$ excitation as in GaN: Er electroluminescence devices. All samples showed characteristic $\mathrm{Er}^{3+} \mathrm{PL}$ centered at $1.54 \mu \mathrm{m}$, which can be assigned to the intra- $4 \mathrm{f} \mathrm{Er}^{3+}$ transition ${ }^{4} \mathrm{I}_{13 / 2^{---}}{ }^{4} \mathrm{I}_{15 / 2}$. The $\mathrm{Er}^{3+}$ 
PL spectra from in-situ Er doped GaN and AlN are very similar. They exhibit nearly featureless spectra (25-35nm @FWHM) suggesting a homogenous distribution of Er sites as typically observed from Er doped glasses. ${ }^{22,23}$ Er peaks located at $1.517 \mu \mathrm{m}, 1.540 \mu \mathrm{m}, 1.548 \mu \mathrm{m}$, and 1.555 $\mu \mathrm{m}$ are either due to Stark splittings and/or multiple Er sites. The overall spectral width of the $\mathrm{Er}^{3+}$ PL from Er implanted GaN was significantly larger (100nm@FWHM) than that observed from Er doped GaN and AlN. Moreover, Er implanted GaN exhibits a more sharply structured $\mathrm{Er}^{3+} \mathrm{PL}$ than the in-situ Er doped III-Nitride samples. Based on previously published data 9,10,11,12,13 , the more complex and broad $\mathrm{Er}^{3+} \mathrm{PL}$ seems to be characteristic for Er implanted GaN samples and is due to a combination of different Er sites and Stark splittings of Er ions in low symmetry sites. For example, Kim et al. identified up to $4 \mathrm{Er}$ sites in Er implanted GaN grown by MOCVD. ${ }^{12}$ The authors demonstrated that it was possible to selectively excite these four sites by three different below-gap absorption processes and through direct intra-4f excitation. Important for future device development was the observation that only a small fraction of incorporated $\mathrm{Er}^{3+}$ ions are excitable through carrier-mediated processes. ${ }^{24}$ Time-resolved PL studies of in-situ Er doped AIN (MOMBE) have indicated the existence of at least two different classes of $\mathrm{Er}^{3+}$ sites with distinct lifetime and excitation schemes. ${ }^{25}$ These results underline that it is crucial to identify the incorporation and excitation mechanism of $\mathrm{Er}^{3+}$ in different sites in order to optimize the material preparation for device applications.

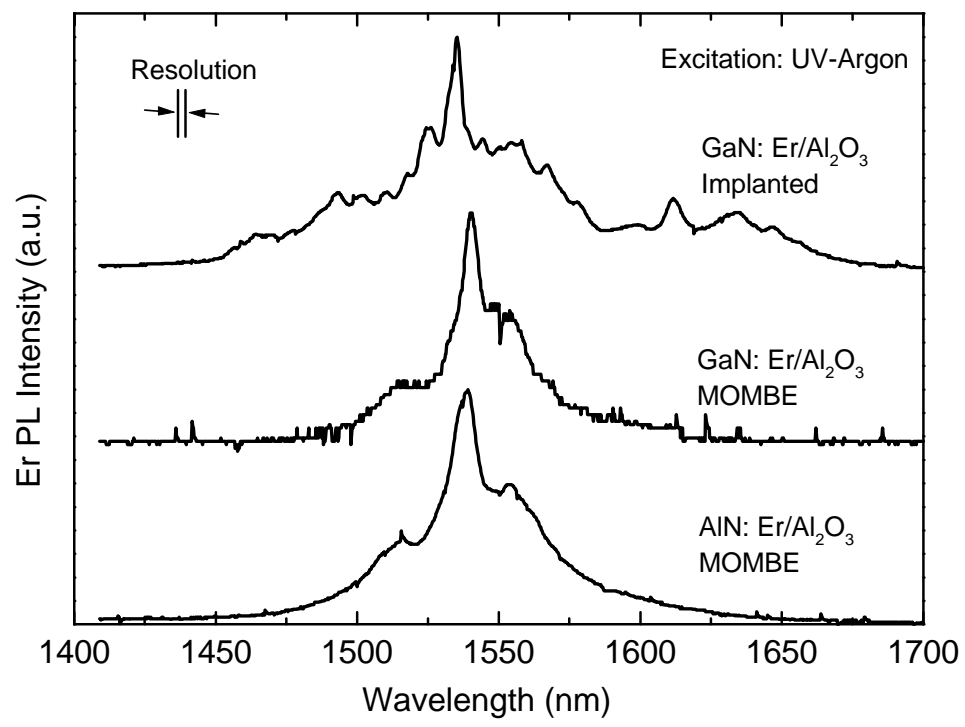

Figure 1: Room-temperature $\mathrm{Er}^{3+} \mathrm{PL}$ spectra of Er implanted GaN and in-situ Er doped GaN and AlN.

Figure 2 depicts a comparison of the absolute $\mathrm{Er}^{3+} \mathrm{PL}$ intensities of GaN: $\mathrm{Er}$ (MOMBE) for below and above-gap excitation at room-temperature. Under below-gap excitation, GaN: Er (TEGa) with high oxygen and carbon background $\left([\mathrm{O}] \sim 10^{20} \mathrm{~cm}^{-3},[\mathrm{C}] \sim 10^{21} \mathrm{~cm}^{-3}\right.$ ) showed an $\mathrm{Er}^{3+}$ PL peak intensity two orders of magnitude larger than $\mathrm{GaN}$ : $\mathrm{Er}(\mathrm{Ga})$ with low $\mathrm{O}$ and $\mathrm{C}$ backgrounds $\left(<10^{19} \mathrm{~cm}^{-3}\right)$. It is interesting to note that also $\mathrm{GaN}$ : Er/Si showed strong $1.54 \mu \mathrm{m}$ PL at room temperature, which makes this material attractive for integration with $\mathrm{Si}$ based optoelectronics. ${ }^{21}$ Drastic improvements of the absolute $\mathrm{Er}^{3+} \mathrm{PL}$ intensity have been observed for Er and oxygen codoped Si and GaAs. The enhanced $1.54 \mu \mathrm{m}$ PL was attributed to an increased concentration of optically active $\mathrm{Er}^{3+}$ ions and a more efficient $\mathrm{Er}^{3+} \mathrm{PL}_{\text {excitation. }}{ }^{26,27,28,29,30}$ Our results for below-gap $\mathrm{Er}^{3+}$ excitation in $\mathrm{GaN}$ suggest that the incorporation of $\mathrm{O}$ and $\mathrm{C}$ introduces 
beneficial mid-gap states that provide efficient energy transfer pathways for $\mathrm{Er}^{3+}$ in GaN. Support for this idea was obtained from photoluminescence excitation (PLE) studies shown in figure 3. In PLE the effectiveness of stimulating $1.54 \mu \mathrm{m} \mathrm{Er}^{3+} \mathrm{PL}$ is measured as a function of excitation wavelength. The PLE spectra of GaN: Er/Si (TEGa) reveal that the incorporation of high $\mathrm{O}$ and $\mathrm{C}$ backgrounds leads to a broad PLE band extending over the entire visible region ( 400-800 $\mathrm{nm}$ ). The GaN: $\mathrm{Er}(\mathrm{Ga})$ sample with low $\mathrm{C}$ and $\mathrm{O}$ content did not show this broad PLE band, but direct intra-4f Er transitions (see e.g. $\sim 525 \mathrm{~nm}$ ). Therefore, the visible output from an Argon-Ion laser leads to a significantly more efficient excitation of $\mathrm{Er}^{3+}$ in $\mathrm{GaN}$ : $\mathrm{Er} / \mathrm{Si}$ (TEGa) than in GaN: $\mathrm{Er} / \mathrm{Si}(\mathrm{Ga})$.
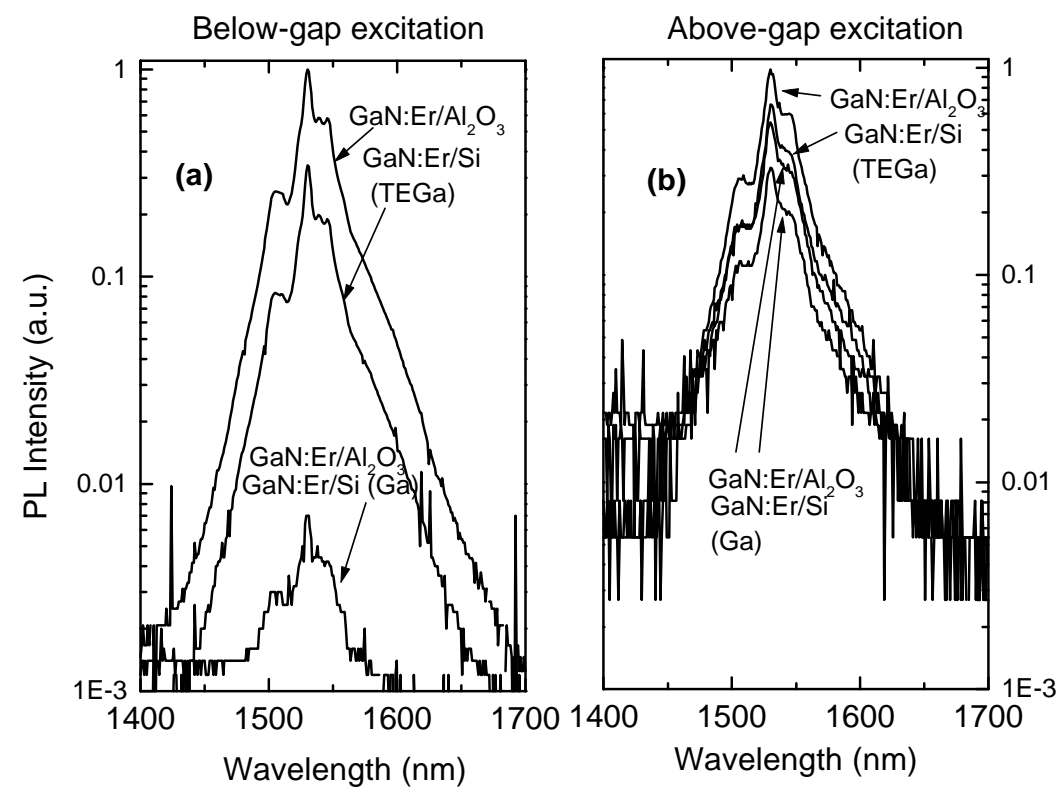

Figure 2: Comparison of $\mathrm{Er}^{3+} \mathrm{PL}$ intensity from Er doped GaN (MOMBE) for a) below-gap and (b) above-gap excitation (300K).

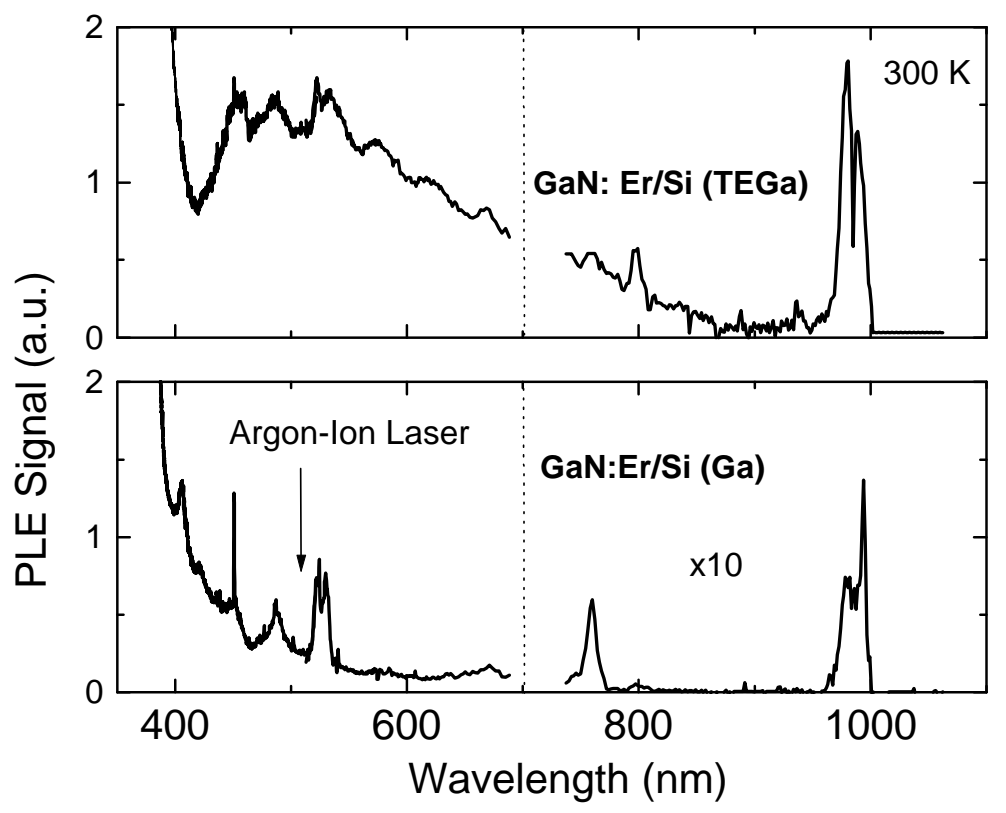

Figure 3: PLE of GaN: Er/Si samples with varying oxygen and carbon content. The incorporation of high oxygen and carbon backgrounds (upper graph) leads to a broad $\mathrm{Er}^{3+}$ excitation band covering the entire visible region. 
Under above-gap excitation (figure 2b) all GaN: Er (MOMBE) samples showed a greatly reduced $\mathrm{Er}^{3+} \mathrm{PL}$ intensity relative to below-gap excitation, independent of carbon and oxygen concentration. A similar PL reduction was reported for Er implanted GaN. ${ }^{31}$ It is somewhat surprising that under above-gap excitation the $\mathrm{Er}^{3+} \mathrm{PL}$ intensities of samples with high oxygen and carbon backgrounds is only roughly twice as strong as the $\mathrm{Er}^{3+} \mathrm{PL}$ observed from low $\mathrm{O}$ and $\mathrm{C}$ contents. These observations suggest that either only a fraction of optically active $\mathrm{Er}^{3+}$ ions are excited and/or the excitation efficiency is reduced under above-excitation. Moreover, it also indicates that high oxygen and carbon concentrations do not necessary lead to an enhanced luminescence for carrier-mediated $\mathrm{Er}^{3+}$ excitation. More work is necessary to address this issue in more detail.

\section{b) Er PL quenching and lifetime}

Figure 4 shows the integrated $\mathrm{Er}^{3+} \mathrm{PL}$ intensity of $\mathrm{Er}$ doped $\mathrm{GaN}$ samples for the temperature range from $15-500 \mathrm{~K}$. The $\mathrm{Er}^{3+} \mathrm{PL}$ was excited either with the broad-band visible (Fig. 4a) or uv (Fig. 4b) outputs of an Argon ion laser corresponding to below and above-gap $\mathrm{Er}^{3+}$ excitation. Under below-gap excitation a significantly different $\mathrm{Er}^{3+} \mathrm{PL}_{\text {quenching behavior }}$ was observed for samples with varying $\mathrm{O}$ and $\mathrm{C}$ contents (see Figure 4a). As was previously observed for $\mathrm{Er}$ and oxygen codoped $\mathrm{Si}$ and $\mathrm{GaAs}$, GaN: $\mathrm{Er}$ samples with high $\mathrm{O}$ and $\mathrm{C}$ concentrations showed a significantly reduced $\mathrm{Er}^{3+} \mathrm{PL}$ quenching compared to samples with low $\mathrm{C}$ and $\mathrm{O}$ backgrounds. ${ }^{26,27,28,29,30}$ Between $15 \mathrm{~K}$ and room-temperature samples with high $\mathrm{O}$ and $\mathrm{C}$ backgrounds showed $\mathrm{Er}^{3+} \mathrm{PL}$ quenching of only $20 \%$, whereas the $1.54 \mu \mathrm{m}$ luminescence from low $\mathrm{O}$ and $\mathrm{C}$ concentration samples decreased by $\sim 90 \%$ over the same temperature range. At higher temperatures also the $\mathrm{O}$ and $\mathrm{C}$ rich samples showed an enhanced $\mathrm{Er}^{3+} \mathrm{PL}$ quenching and the luminescence decreased to roughly $10 \%$ of their low temperature value at $500 \mathrm{~K}$. Under above-gap excitation the situation was different. As shown in Fig. 4(b) hardly any difference in $\mathrm{Er}^{3+}$ PL quenching behavior was observed for samples with varying oxygen/carbon content. As mentioned above, the oxygen and carbon concentration does not greatly effect the carriermediated $\mathrm{Er}^{3+}$ excitation. Up to room temperature the $\mathrm{Er}^{3+} \mathrm{PL}$ decreased for all four samples by less than $50 \%$ relative to its low temperature value. When heating the samples above room temperature, we observed a steady decrease of the $\mathrm{Er}^{3+} \mathrm{PL}$ with increasing temperature. At 550 $\mathrm{K}$, the $\mathrm{Er}^{3+} \mathrm{PL}$ from all samples had decreased by nearly $90 \%$ of its low temperature value.

To gain more insight in the observed $\mathrm{Er}^{3+} \mathrm{PL}$ quenching, we performed initial luminescence lifetime studies on the two oxygen and carbon rich samples, GaN: Er/Si (TEGa) and GaN:Er/Sapphire (TEGa). These samples showed the strongest $\mathrm{Er}^{3+} \mathrm{PL}$ signal, which allowed us to carry out lifetime studies for the entire range $15-500 \mathrm{~K}$. It was observed that the $\mathrm{Er}^{3+} \mathrm{PL}$ decay curves were non-exponential which provides further support for the existence of a wide distribution of $\mathrm{Er}^{3+}$ sites. To describe the luminescence decay an average lifetime $\langle\tau\rangle$ was used:

$$
<\tau>=\frac{\int I(t) t d t}{\int I(t) d t}
$$

where $\mathrm{I}(\mathrm{t})$ is the luminescence decay curve and $\mathrm{t}$ is the time. ${ }^{32,33}$ The low temperature lifetime of both samples was found to be $\sim 130 \mu$ s and decreased to $\sim 100 \mu$ s at room temperature. At $500 \mathrm{~K}$ the lifetime had shortened to $\sim 50 \mu \mathrm{s}$. These lifetimes are significantly shorter than previously reported lifetimes for Er implanted GaN, which were in the order of $1-2 \mathrm{~ms} .{ }^{10,16}$ It is possible that the short lifetimes are due to a higher oscillator strength of $^{\mathrm{Er}^{3+}}$ transitions in MOMBE grown samples compared to Er implanted GaN. In order to confirm this explanation direct absorption measurements of intra- $4 \mathrm{Er}$ transitions are necessary. It is also conceivable that the $\mathrm{Er}^{3+}$ lifetimes 
are shortened due intra- $4 \mathrm{f}$ non-radiative decay or cooperative processes such as upconversion or energy migration. ${ }^{34}$ Power dependent lifetime studies, however, did not show any change in lifetime with increasing excitation density suggesting that the later processes are not dominant in $\mathrm{GaN}: \mathrm{Er}$.
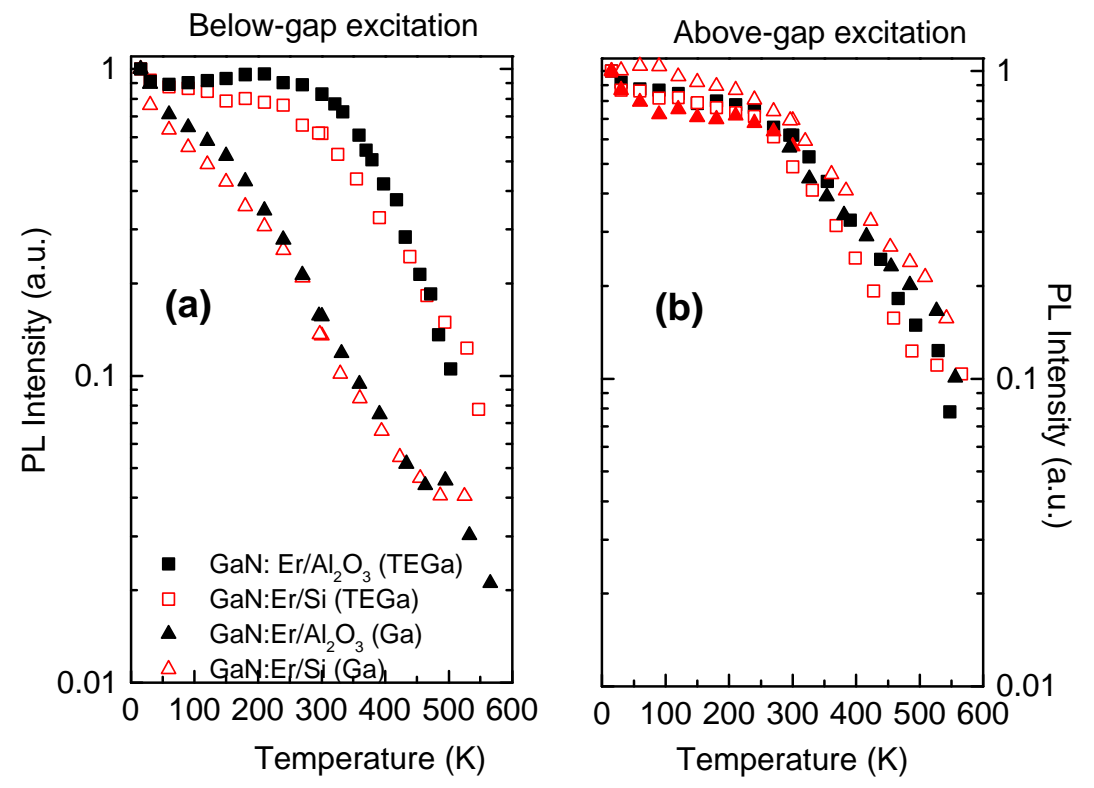

Figure 4: Temperature dependence of the integrated PL intensity from in-situ Er doped GaN for above and below gap excitation.

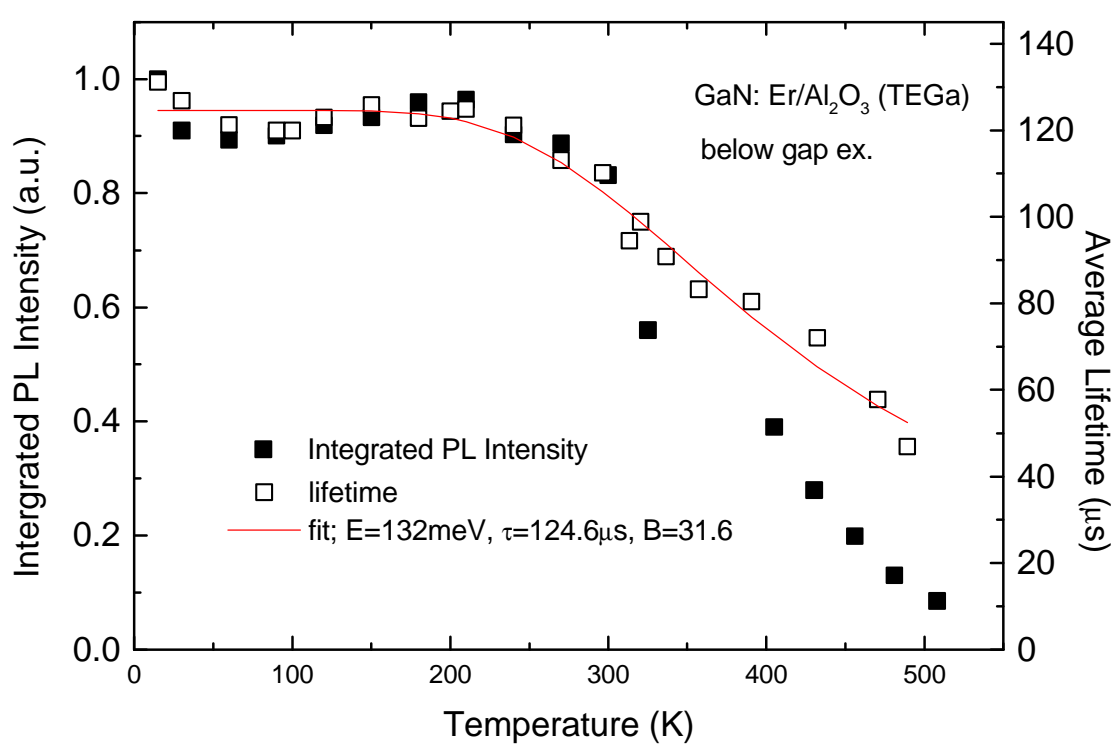

Figure 5: Comparison of the temperature dependence of $\mathrm{Er}^{3+}$ intergrated PL and lifetime for in-situ Er doped GaN.

It is instructive to compare the temperature dependence of PL intensity and lifetime as shown in figure 5. In the following discussion it is assumed that the $\mathrm{Er}^{3+} \mathrm{PL}$ intensity is proportional to the number (or concentration) of excited $\mathrm{Er}$ ion $\left(\mathrm{N}_{\mathrm{Er}}{ }^{*}\right)$ and the radiative decay rate $\left(\mathrm{w}_{\mathrm{rad}}\right)$. Under steady state condition and low pump power intensities the $\mathrm{Er}^{3+} \mathrm{PL}$ signal can be written as: 


$$
I_{P L}=C \cdot \frac{I_{p}}{h c} \cdot \lambda_{p} \cdot \sigma_{e x} \cdot N_{t o t} \cdot w_{r a d} \cdot \tau
$$

where $C$ is a constant, $I_{p}$ is the pump intensity, $\lambda_{\mathrm{p}}$ is the pump wavelength, $\sigma_{\mathrm{ex}}$ is the excitation cross-section defined as the product of pump efficiency and absorption cross-section, $N_{\text {tot }}$ is the total Er concentration, $w_{\text {rad }}$ is the radiative decay rate, and $\tau$ is the luminescence lifetime. Under the assumption that the radiative decay rate and total $\mathrm{Er}$ concentrations are temperature independent, the $\mathrm{Er}^{3+}$ PL quenching at higher temperatures can be explained by either the onset of nonradiative deexcitation processes reducing the $\mathrm{Er}^{3+}$ lifetime and/or by a decrease of the excitation efficiency.

The comparison of the temperature dependent $\mathrm{Er}^{3+} \mathrm{PL}$ intensity and lifetime enables one to separate the above mentioned $\mathrm{Er}^{3+} \mathrm{PL}$ quenching mechanism. As can be inferred from figure 5, the $\mathrm{Er}^{3+}$ lifetime follows the change in integrated PL intensity very closely up to room temperature. This means that between 15 and $300 \mathrm{~K}$ the small decrease in $\mathrm{Er}^{3+}$ PL intensity is due to nonradiative decay. Above room-temperature the lifetime changes less than the integrated $\mathrm{Er}^{3+}$ PL intensity. This suggests that $\mathrm{Er}^{3+} \mathrm{PL}$ quenching occurring above room temperature is not only due nonradiative deexcitation, but also due to a reduction in excitation efficiency. Nearly the same $\mathrm{Er}^{3+} \mathrm{PL}$ intensity and lifetime behavior was observed for $\mathrm{GaN}$ : $\mathrm{Er} / \mathrm{Si}$ (not shown). Similar to other Er doped semiconductor systems, we assume that the excited Er ion can decay either through emission of a $1.54 \mu \mathrm{m}$ photon or through non-radiative energy transfer processes to the host (also referred to as "backtransfer" ${ }^{28,35}$ ). It is further assumed that internal $\mathrm{Er}^{3+}$ quantum efficiency does not change with temperature. The change in lifetime is then described by a thermally activated nonradiative decay process, which is controlled by Boltzmann's law:

$$
\frac{1}{\tau}=\frac{1}{\tau_{r a d}}\left(1+B \cdot \exp \left(-\frac{E}{k T}\right)\right)
$$

where $\tau_{\text {rad }}$ is the radiative lifetime, $\mathrm{E}$ is the thermal activation or threshold energy for nonradiative decay, and $\mathrm{B}$ is a constant. Fitting equation 3 to the lifetime data shown in figure 5 gives the following parameter values: $\tau_{\mathrm{rad}}=124.6 \mu \mathrm{s}, \mathrm{B}=31.6$, and an activation energy $\mathrm{E}=132 \mathrm{meV}$. The fit result suggests the existence of a defect-related level close to $\mathrm{Er}^{3+}$, which acts as a particular effective channel for nonradiative energy transfer between $\mathrm{Er}^{3+}$ and the host. The deexcitation of Er ions through some mid-gap level has been discussed before for Er doped $\mathrm{Si}$ and GaAs. In the backtransfer model as proposed by Takahei et al. ${ }^{28}$, the existence of an Errelated level provides the possibility of energy backtransfer where excited Er ions transfer an excited carrier back to the defect level followed by a thermalization into the conduction band. This model for the deactivation of excited $\mathrm{Er}^{3+}$ ions may also be relevant for GaN:Er, however, no experimental evidence for an Er-related defect level has yet been reported. A more detailed comparison of the $\mathrm{Er}^{3+}$ lifetimes of $\mathrm{GaN}$ : Er samples with varying oxygen and carbon content is in progress and promises to provide deeper insight in the Er excitation and deexcitation mechanisms.

\section{c) visible PL from GaN: Er}

Very recently green luminescence was observed from $\mathrm{GaN}$ : $\mathrm{Er}$ grown by solid source molecular beam epitaxy under above-gap excitation. ${ }^{36}$ Narrow lines centered at $537 \mathrm{~nm}$ and $558 \mathrm{~nm}$ were identified to be due to the intra-4f Er transitions ${ }^{2} \mathrm{H}_{11 / 2} \rightarrow{ }^{4} \mathrm{I}_{15 / 2}$ and ${ }^{4} \mathrm{~S}_{3 / 2} \rightarrow{ }^{4} \mathrm{I}_{15 / 2}$. Subsequently the same authors reported green electroluminescence from Er doped GaN Schottky 
barrier diodes. ${ }^{37}$ In figure 6 we show the visible PL spectra of GaN: Er/Sapphire prepared by MOMBE. We observed no green emission lines from any of the investigated samples under below $(488 \mathrm{~nm})$ or above-gap $(325 \mathrm{~nm})$ excitation. Interestingly, however, for below-gap excitation we observed a broad red luminescence band, which showed sharp structure at around $665 \mathrm{~nm}$ and $690 \mathrm{~nm}$. This emission was not found for the GaN: Er/Si samples. Preliminary studies indicated that the peaks at $665 \mathrm{~nm}$ and $690 \mathrm{~nm}$ had lifetimes in the millisecond range. The peak at $665 \mathrm{~nm}$ is assigned to the ${ }^{4} \mathrm{~F}_{9 / 2} \rightarrow{ }^{4} \mathrm{I}_{15 / 2}$ transition of $\mathrm{Er}^{3+}$ ions, whereas the origin of the $690 \mathrm{~nm}$ peak is not yet know. The data suggest that the $690 \mathrm{~nm}$ line is a pure electronic transition (no-phonon line) accompanied by a vibronic sideband at around $720 \mathrm{~nm}$. A more detailed study is currently being undertaken to identify the origin of the red luminescence from GaN:Er/Sapphire.

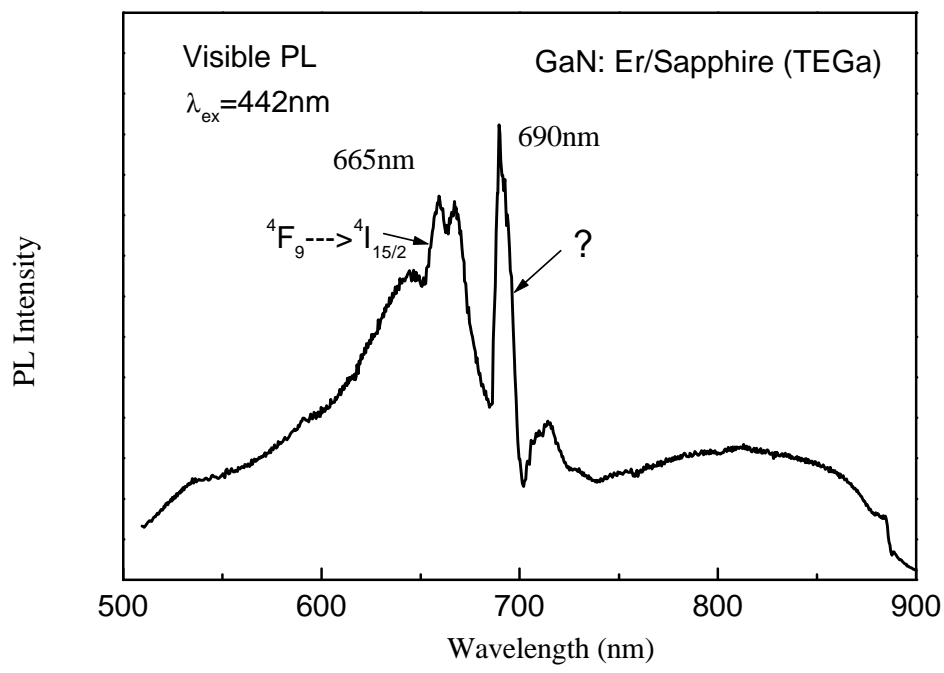

Figure 6: Visible PL spectrum of GaN: Er/Sapphire for below-gap excitation at roomtemperature.

\section{SUMMARY}

We presented new results on the $1.54 \mu \mathrm{m}$ luminescence properties of GaN: Er grown by MOMBE. It was observed that high oxygen and carbon concentrations $\left(\sim 10^{20} \mathrm{~cm}^{-3}\right)$ enhance the $1.54 \mu \mathrm{m} \mathrm{Er}^{3+} \mathrm{PL}$ intensity and reduce the $\mathrm{Er}^{3+} \mathrm{PL}$ quenching under below-gap excitation. Moreover, PLE studies revealed that oxygen/carbon introduces a broad below-gap PLE band, which provides an efficient pathway for $\mathrm{Er}^{3+}$ excitation. A direct comparison of the temperature dependence of $\mathrm{Er}^{3+} \mathrm{PL}$ intensity and lifetime suggests that both nonradiative decay and a decrease in excitation efficiency reduce the $\mathrm{Er}^{3+} \mathrm{PL}$. Under above-gap excitation the absolute $\mathrm{Er}^{3+} \mathrm{PL}$ intensity from all samples was greatly reduced and only a weak PL dependence on the oxygen and carbon concentration was observed. The weak above-gap $\mathrm{Er}^{3+} \mathrm{PL}$ suggests that the efficiency of electroluminescence devices (forward biased LED's) based on currently available materials will be rather low. More materials engineering will be necessary to optimize the $1.54 \mu \mathrm{m} \mathrm{Er}^{3+}$ PL from GaN: Er. Initial results of visible luminescence studies were also presented. It was found that only under below-gap excitation $\mathrm{GaN}$ :Er on sapphire emits red luminescence peaking at 665 and $690 \mathrm{~nm}$. The $665 \mathrm{~nm}$ line was assigned to an intra- $4 \mathrm{f} \mathrm{Er}^{3+}$ transition, whereas 
the origin of the $690 \mathrm{~nm}$ line is not yet known. More detailed studies on the luminescence properties of $\mathrm{GaN}$ : Er are in progress in will be presented in a forthcoming paper.

\section{ACKNOWLEDGMENTS}

We would like to thank George Ofori-Boudu and Jakeithia Preajan for the help with some PL experiments. The authors from Hampton University acknowledge financial support by NASA through Grant NCC-1-251 and the Army Research Office through Grants DAAH04-96-1-0089 and DAAG55-98-1-0112. The work at the University of Florida was supported by ARO grant DAAH04-96-1-0089.

\section{REFERENCES}

R. L. Bell, J. Appl. Phys. Comm. 34, 1563 (1963).

2 H. Ennen, U. Kaufmann, G. S. Pomrenke, J. Schneider, J. Windschif, and A. Axmann, J. Crystal Growth 64, 165 (1983).

3 G. S. Pomrenke, P. B. Klein, and D. W. Langer, Rare Earth Doped Semiconductors, Material Research Society Symposium Proceedings, Vol. 301, Material Research Society, Pittsburg, 1993.

4 S. Coffa, A. Polman, and R.N. Schwartz, Rare Earth doped Semiconductors II, Materials Research Society Symposium Proceedings, Vol. 422, Material Research Society, Pittsburgh, PA, 1996.

5 P. N. Favennec, H.L. Haridon, M. Salvi, D. Moutonnet, and Y. Le Guillou, Electr. Lett. 25, 718 (1989).

6 A.J. Neuhalfen and B.W. Wessels, Appl. Phys. Lett. 60, 2657 (1992).

7 W. J. Choyke, R. P. Devaty, L. L. Clemen, M. Yoganathan, G. Pensl, and Ch. Hässler, Appl. Phys. Lett. 65, 1668 (1994).

8 J. M. Zavada and D. Zhang, Solid-State Electronics, Vol. 38, No.7, 1285 (1995).

9 R. G. Wilson, R. N. Schwartz, C. R. Abernathy, S. J. Pearton, N. Newman, M. Rubin, T. Fu, and J. M. Zavada, Appl. Phys. Lett. 65, 992 (1994).

10 C.H. Qui, M. W. Leksono, J. I. Pankove, J. T. Torvik, R. J. Feuerstein, and F. Namavar, Appl. Phys. Lett. 66, 562 (1995).

11 E. Silkowski, Y.K. Yeo, R. L. Hengehold, B. Goldenberg, and G. S. Pomrenke, MRS Proceedings, Vol. 422, 69 (1996).

12 S. Kim, S. J. Rhee, D. A. Turnbull, E. E. Reuter, X. Li, J. J. Coleman, and S. G. Bishop, Appl. Phys. Lett. 71, 231 (1997).

13 Myo Thaik, U. Hömmerich, R. N. Schwartz, R. G. Wilson, and J.M. Zavada, Appl. Phys. Lett. 71, 2641 (1997).

14 J. D. MacKenzie, C. R. Abernathy, S. J. Pearton, U. Hömmerich, X. Wu, R. N. Schwartz, R. G. Wilson, J. M. Zavada, Appl. Phys. Lett. 69, 2083 (1996).

15 J. D. MacKenzie, C. R. Abernathy, S. J. Pearton, U. Hömmerich, X. Wu, R. N. Schwartz, R. G. Wilson, J. M. Zavada, J. Cryst. Growth 175/176, 84 (1997).

16 D.M. Hansen, R. Zhang, N. R. Perkins, S. Safvi, L. Zhang, K.L. Bray, and T. F. Kuech, Appl. Phys. Lett 72, 1244 (1998).

17 A. J. Steckl and R. Birkhahn, Appl. Phys. Lett. 73, 1701 (1998).

18 A. J. Steckl, M. Garter, R. Birkhahn, and J. Scofield, Appl. Phys. Lett. 73, 2450, (1998).

19 J. T. Torvik, R. J. Feuerstein, C. H. Qui, J. I. Pankove, and F. Namavar, J. Appl. Phys. 69 2098 (1996). 
${ }^{20}$ H. Shen, J. Pamulapati, J. D. Mackenzie, F. Ren, C. R. Abernathy, and J. M. Zavada, MRS Fall Meeting 1998, paper G11.5.

21 J. D. MacKenzie, C. R. Abernathy, S. J. Pearton, U. Hömmerich, J. T. Seo, R. G. Wilson, and J. M. Zavada, Appl. Phys. Lett. 72, 2710 (1998).

22 W. J. Miniscalco, J. of Lightwave Techn. 9, 234 (1991).

23 B. J. Ainslie, J. of Lightwave Techn. 9, 220 (1991).

24 S. J. Rhee, S. Kim, X. Li, J. J. Coleman, and S. G. Bishop, Mat. Res. Soc. Symp. Proc., Vol. 482, 667 (1998).

25 X. Wu, U. Hömmerich, J. D. MacKenzie, C. R. Abernathy, S. J. Pearton, R. N. Schwartz, R. G. Wilson, and J. M. Zavada, Appl. Phys. Lett. 70, 2126 (1997).

26 J. L. Benton, D.J. Eaglesham, M. Almonte, P.H. Citrin, M. A. Marcus, D.L. Adler, D. C. Jacobson, and J. M. Poate, Mat. Res. Soc. Symp. Proc., Vo. 301, 119 (1993).

27 S. Coffa, F. Priolo, G. Franzo, V. Bellani, A. Carnera, C. Spinella, Phys. Rev. B, 48, 11782 (1993).

28 K. Takahei, A. Taguchi, J. Appl. Phys. 74, 1979 (1993).

29 J. Michel, J.L. Benton, R.F.Ferrante, D.C. Jacobson, D.J.Eaglesham, E.A. Fitzgerald, Y.H. Xie, J.M. Poate, and L.C. Kimmerling, J. Appl. Phys. 70, 2672 (1991).

30 S. Coffa, F. Priolo, G. Franzo, V. Bellani, A. Carnera, and C. Spinella, Mat. Res. Soc. Symp. Proc., Vol. 301, 125, 1993.

31 J. T. Torvik, R. J. Feuerstein, C. H. Qui, J. I. Pankove, and F. Namavar, Mat. Res. Soc. Symp. Proc., Vol. 482, 579, 1998.

32 J. Fernandez, R. Balda, M. A. Illarramendi, G. F. Imbusch, J. Lumin. 58, 294 (1994).

33 L. J. Andrews, A. Lempicki, and B. C. McCollum, J. Chem. Phys. 74, 5526 (1981).

34 G. N. van den Hoven, Jung H. Shin, A. Polman, S. Lombardo, and S. U. Campisano, J. Appl. Phys. 78, 2642, 1995.

35 P. G. Kik, M. J. A de Dood, K. Kikoin and A. Polman, Appl. Phys. Lett. 70, 1721 (1997).

36 R. Birkhahn and A.J. Steckl, Appl. Phys. Lett. 73, 2143 (1998).

37 A. J. Steckl, M. Garter, R. Birkhahn, and J. Scofield, Appl. Phys. Lett. 73, 2450, (1998). 